\title{
Genetic Rat Models of Parkinson's Disease
}

\author{
Ryan M. Welchko, ${ }^{1}$ Xavier T. Lévêque, ${ }^{2}$ and Gary L. Dunbar ${ }^{3,4}$ \\ ${ }^{1}$ Field Neurosciences Institute, Laboratory for Restorative Neurology, Department of Psychology, Central Michigan University, \\ Mt. Pleasant, MI 48859, USA \\ ${ }^{2}$ Field Neurosciences Institute, Laboratory for Restorative Neurology, Program in Neuroscience, Central Michigan University, \\ Mt. Pleasant, MI 48859, USA \\ ${ }^{3}$ Field Neurosciences Institute, Laboratory for Restorative Neurology, Department of Psychology and Program in Neuroscience, \\ College of Medicine, Central Michigan University, Mt. Pleasant, MI 48859, USA \\ ${ }^{4}$ Field Neurosciences Institute, St. Mary's of Michigan, Saginaw MI 48604, USA
}

Correspondence should be addressed to Gary L.Dunbar, dunba1g@cmich.edu

Received 10 December 2011; Accepted 20 January 2012

Academic Editor: Kah-Leong LIM

Copyright (C) 2012 Ryan M. Welchko et al. This is an open access article distributed under the Creative Commons Attribution License, which permits unrestricted use, distribution, and reproduction in any medium, provided the original work is properly cited.

\begin{abstract}
Parkinson's disease (PD) is a neurodegenerative disease characterized by a specific loss of dopaminergic neurons. Although the vast majority of PD cases are idiopathic in nature, there is a subset that contains genetic links. Of the genes that have been linked to $\mathrm{PD}, \alpha$-synuclein and leucine-rich repeat kinase 2 have been used to develop transgenic rat models of the disease. In this paper we focused on the various transgenic rat models of PD in terms of their ability to mimic key symptoms of PD in a progressive manner. In general, we found that most of these models provided useful tools for the early stages of PD, but the development of new transgenic rats that present significant neuropathologic and motoric deficits in a progressive manner that more accurately mimics PD is needed.
\end{abstract}

\section{Introduction}

Parkinson's disease (PD) is the second most common neurodegenerative disorder. It is hypothesized that PD is caused by a combination of genetic and environmental factors. A specific loss of dopaminergic (DA) neurons in the substantia nigra $(\mathrm{SN})$ in $\mathrm{PD}$ results in the significant loss of DA innervation of the neostriatum via the nigrostriatal pathway. The progressive loss of this DA innervation, in turn, is responsible for the characteristic motor symptoms of $\mathrm{PD}$, which include akinesia, resting tremors, and postural instability.

A hallmark histopathological marker for PD is the presence of cytoplasmic inclusions, known as Lewy bodies (LBs), which are found throughout the mesencephalon of the PD brain, including the substantia nigra [1]. The major component of LBs is the presynaptic protein called $\alpha$ synuclein ( $\alpha$-syn) [2]. To date, it is not known whether the mechanisms underlying the formation of LBs are the cause or a consequence of the disease. Some familial forms of PD have been linked to mutations in genes that code for $\alpha$-syn, as well as genes that code for leucine-rich repeat kinase 2 (LRRK2), DJ-1, and Parkin [3, 4]. These mutations have formed the basis of developing transgenic rodent models of PD.

Presently, the only treatments for PD are palliative in nature and usually utilize dopamine agonists, such as levodopa (L-DOPA). Surgical techniques, such as pallidotomies and deep-brain stimulation, have also proved to be successful in reducing some of the major motor symptoms of PD. To date, the most promising avenue of research aimed at restoring lost function in PD appears to be cell therapies, and much of the recent research efforts in this domain have focused on use of stem cells.

However, significant advances in developing effective therapies for PD have been limited by the lack of a valid animal model that closely mimic the progressive neuropathology and behavioral deficits of PD. Most research on $\mathrm{PD}$ has utilized rodents that are given specific neurotoxins, including 6-hydroxydopamine (6-OHDA), 1-methyl4-phenyl-1,2,3,6-tetrahydropyridine (MPTP), paraquat, and 
TABLE 1: Summary of studies using genetic rat models of PD. Note that \% cell loss SNpc is percentage of cell loss in the substantia nigra, pars compacta, relative to number of cells in the intact, untreated controls; "n.a." indicates data for percentage loss of SNpc cells was not available; plus sign (+) indicates significant differences and "n.s." indicates non-significant differences between genetically altered rats and wild-type controls on measures of protein aggregation or behavioral deficits (i.e., stimulant-induced rotational activity, paw placement in cylinder task, and/or spontaneous motor activity in open-field).

\begin{tabular}{|c|c|c|c|c|c|}
\hline Gene & Method & $\begin{array}{l}\text { Percentage of } \\
\text { cell loss SNpc }\end{array}$ & $\begin{array}{c}\text { Protein } \\
\text { aggregation }\end{array}$ & Behavior deficit & Author year \\
\hline h $\alpha$-syn, $\alpha$-syn A53T & rAAV & $30 \%-80 \%$ & + & $+($ in $25 \%$ of animals $)$ & Kirik et al. 2002 [12] \\
\hline h $\alpha$-syn A30P & rAAV & $53 \%$ & + & n.s. & Klein et al. 2002 [13] \\
\hline h $\alpha$-syn A30P & HIV-1 Lentivirus & $33 \%$ & + & None performed & Lo Bianco et al. 2002 [14] \\
\hline h $\alpha$-syn A53T & HIV-1 Lentivirus & $24 \%$ & + & None performed & Lo Bianco et al. 2002 [14] \\
\hline h $\alpha$-syn & rAAV & $50 \%$ & + & n.s. & Yamada et al. 2004 [15] \\
\hline h $\alpha$-syn & $\mathrm{rAAV} 2 / 5$ & $40-60 \%$ & + & None performed & Gorbatyuk etal. 2008 [16] \\
\hline h $\alpha$-syn S129A & $\mathrm{rAAV} 2 / 5$ & $66-70 \%$ & + & None performed & Gorbatyuk etal. 2008 [16] \\
\hline h $\alpha$-syn S129D & $\mathrm{rAAV} 2 / 5$ & n.a. & + & None Performed & Gorbatyuk et al. 2008 [16] \\
\hline h $\alpha$-syn & $\mathrm{rAAV} 2 / 6$ & $11-22 \%$ & + & None Performed & da Silveira et al. 2009 [17] \\
\hline h $\alpha$-syn A30P & $\mathrm{rAAV} 2 / 6$ & $11-22 \%$ & + & None Performed & da Silveira et al. 2009 [17] \\
\hline h $\alpha$-syn WT S129A & rAAV2/6 & over $70 \%$ & + & None Performed & da Silveira et al. 2009 [17] \\
\hline h $\alpha$-syn WT S129D & $\mathrm{rAAV} 2 / 6$ & n.a. & + & None Performed & da Silveira et al. 2009 [17] \\
\hline h $\alpha$-syn A30P S129A & $\mathrm{rAAV} 2 / 6$ & over $70 \%$ & + & None Performed & da Silveira et al. 2009 [17] \\
\hline h $\alpha$-syn A30P S129D & $\mathrm{rAAV} 2 / 6$ & n.s. & + & None Performed & da Silveira et al. 2009 [17] \\
\hline h $\alpha$-syn A30P & TAT & $21.3-31.3 \%$ & n.s. & + & Recchia et al. 2008 [18] \\
\hline h $\alpha$-syn A30P A53T & Microinjection & n.a. & n.s. & + & Lelan et al. 2011 [19] \\
\hline LRRK2 G2019S & Rad & $21.4 \%$ & n.s. & None Performed & Dusonchet et al. 2011 [20] \\
\hline LRRK2 G2019S & TRE-tTA & $0 \%$ & n.s. & + & Zhou et al. 2011 [21] \\
\hline
\end{tabular}

rotenone, that are administrated into the substantia nigra pars compacta $(\mathrm{SNpc})$ or nigrostriatal pathway. Although these models have yielded valuable insights into potentially useful therapies for PD, they do not accurately mimic the full constellation of neuropathological and behavioral deficits associated with PD. Recently, transgenic animal models of PD have been developed by inserting the mutated human gene in rodents. Although most of these transgenic models have been developed in mice, more recent efforts have focused on developing transgenic rat models of PD.

The purpose of this paper is to provide an update on the newly developed genetic rat models of PD and to provide some assessment of their usefulness in therapeutic research. We will attempt to compare each of the newly developed models in terms of their ability to mimic both the neuropathological and behavioral features of the disease (see Table 1). We will also describe some of the early therapeutic research using these models. Although none of these models provide the optimal tool for testing potential treatments for $\mathrm{PD}$, we think that some of these models have the potential to provide an improvement over the more commonly used neurotoxic rat models.

\section{2. $\alpha$-Synuclein Rat Models}

The most thoroughly studied transgenic rat model of PD is the $\alpha$-synuclein rat model. As indicated previously, some familial forms of PD have been linked to $\alpha$-syn, a small neuronal 140 amino acid protein $(14.4 \mathrm{kDa})$ [5], that is located in presynaptic terminals. In humans, the gene for $\alpha$-syn is located on chromosome 4q21-q23 [6] and this protein is highly expressed in the brain. Missense autosomal dominant mutations at A53T [7], E46K [8], and A30P [9] of the gene-coding region of $\alpha$-syn have been linked to LB aggregates and Lewy neurites in patients with rare, early onset PD. The presynaptic presence of $\alpha$-syn suggests a role in synaptic vesicle trafficking. It has been shown that $\alpha$-syn is a dose-dependent inhibitor of tyrosine hydroxylase (TH) [10]. On the other hand, $\alpha$-syn could be a link in the dopamine transporter and, in this way, increase dopamine reuptake [11]. As such, it appears that an optimal level of $\alpha$-syn may be critical for proper functioning of DA neurons.

Transgenic $\alpha$-syn models have been created in adult rats utilizing adenoassociated virus (rAAV) or HIV-1-derived lenti-virus to transduce mutated human $\alpha$-syn genes into the nigrostriatal pathway. Also, HIV TAT-mediated protein transduction has been used to transduce mutated human $\alpha$-syn protein. Although there has been some success with achieving expression of $\alpha$-syn in DA neurons within the $\mathrm{SN}$, the amount of DA neuronal degeneration varied greatly between studies assessed by $\mathrm{TH}$ immunohistochemistry (IHC). Some of this variation could be due to differing forms of the $\alpha$-syn gene used, the method of delivery utilized, or the gene promoter used. For example, Klein et al. [13] found a 53\% reduction of TH-positive DA neurons when they injected rAAV expressing the mutant human $\alpha$-syn A30P into the SN, while Kirik et al. [12] reported a 30-80\% reduction when they injected $\mathrm{rAAV}$ expressing the mutant 
human $\alpha$-syn A53T in the SN. Klein et al. [13] and Kirik et al. utilized a hybrid cytomegalovirus (CMV)/chicken $\beta$-actin (CBA) promoter to drive expression of the differing mutant $\alpha$-syn genes. This specific promoter is the CBA promoter with enhancer elements from the CMV promoter added. In addition, Kirik et al. [12] found a 50\% reduction in $\mathrm{TH}$ positive DA striatal innervation at eight weeks after injection. In term of behavior, Kirik et al. [12] reported that $25 \%$ of the $\alpha$-syn-injected rats were impaired during apomorphineinduced rotation assessments and on a paw-reaching (i.e., cylinder) task, an impairment that is compatible to a loss of more than $60 \%$ of DA SN neurons. However, Klein et al. [13] did not find a significant behavioral impairment in amphetamine-induced rotations. Both Kirik and Klein groups of investigators reported progressive pattern of neurodegeneration that included $\alpha$-syn aggregates that resembled LBs in PD patients. As such, these transgenic rat models of PD more closely mimicked the progressive loss of dopaminergic neurons in PD than do the neurotoxic rat models of this disease.

Yamada et. al [15] created an $\alpha$-syn model using rAAV, but utilized a cytomegalovirus (CMV) promoter which differed from the CMV/CBA promoter used by the Kirik and Klein groups $[12,13]$. Yamada et al. reported a $50 \%$ decrease in the number of DA neurons in the injected $\mathrm{SN}$, but this decrease was insufficient to produce significant differences between the injected rats and controls when these animals were assessed on measures of apomorphine-induced rotation.

Recchia et al. [18] created an $\alpha$-syn model of PD by administering intranigral injections of the A30P mutated form of $\alpha$-syn, fused to a TAT fusion protein. Their study compared unilateral injections of TAT- $\alpha$-syn A30P protein, $\alpha$-syn A30P protein, 6-OHDA, and sham controls into the $\mathrm{SN}$ at various time points. Neurodegeneration and genetic expression were assessed via IHC, western blot, HPLC analysis, and behavioral assessment. They found that the $\alpha$ syn A30P protein, without the TAT fusion protein, did not integrate within neurons of the $\mathrm{SN}$, whereas $80 \%$ of $\mathrm{TH}$ positive cells were found to express $\alpha$-syn A30P following TAT- $\alpha$-syn A30P injection. No formations of LBs or Lewy neurites were found in these animals. The TAT- $\alpha$-syn A30P distribution, assessed by western blot, was found within the $\mathrm{SN}$, striatum, and contralateral cortex at 24 hours after injection. At 15 and 30 days post-injection, the TAT- $\alpha$ syn $\mathrm{A} 30 \mathrm{P}$ produced a $26.3 \pm 5.0 \%$ loss in $\mathrm{TH}$ positive neurons, compared to the $81.2 \pm 2.1 \%$ loss in TH positive neurons following the 6-OHDA injections. At 7 days postinjection, the TAT- $\alpha$-syn A30P animals showed a $20.8 \pm$ $3.6 \%$ reduction in DA and $16.6 \pm 2.5 \%$ reduction in 3,4 dihydroxyphenylacetic acid (DOPAC) within the ipsilateral, compared to the contralateral, striatum. In comparison, the animals receiving 6-OHDA injections showed a $60.6 \pm$ $3.6 \%$ reduction in $\mathrm{DA}$ and a $59.7 \% \pm 4.5$ reduction in DOPAC within the ipsilateral, compared to the contralateral, striatum. Administration of apomorphine did not produce a significant number of ipsilateral rotations in animals receiving TAT- $\alpha$-syn A30P injections. However, rats receiving TAT$\alpha$-syn A30P injections showed increasing motor impairment at day 30 and day 90 when motor function was accessed via rotorod and footprint analysis.

More recently, Sanchez-Guajardo et al. [22] studied the temporal profile of microglia activation in an $\alpha$-syn rat model of PD. They injected rAAV2/5 expressing human wild type (hWT) $\alpha$-syn directly into the right SN. Rats were divided into two groups based upon the concentration of rAAV2/5 hWT $\alpha$-syn. The $\alpha$-syn neurodegeneration group received a lower concentration of $\mathrm{rAAV} 2 / 5 \mathrm{hWT} \alpha$-syn, while the $\alpha$-syn cell-death group received a greater concentration. The $\alpha$-syn neurodegeneration rats did not exhibit a significant reduction in TH-positive neurons within the SN. However, a decrease in TH-positive fiber density within the striatum and pathological formations were observed in these animals. The $\alpha$-syn cell-death group exhibited a significant reduction in TH-positive neurons within the SN and a reduction of TH-positive fiber density within the striatum. An increase in microglia was seen in both groups at four weeks after injection. The activated microglia was significantly higher in the neurodegeneration group at 4 weeks, and the levels of microglia returned to control levels at 8-15 weeks. Within the cell-death group, microglia levels peaked at 8 weeks and returned to control levels at 15 weeks.

Lelan et al. [19] created a transgenic $\alpha$ syn rat utilizing microinjection to transfer the construct pUTHTV expressing the A30P and A53T double-mutated human $\alpha$ syn under the control of the rat $\mathrm{TH}$ promoter. These investigators observed the expression of mutated human $\alpha$-syn in olfactory bulbs and within SN. They also observed an olfactory impairment in mutated human $\alpha$ syn transgenic rats, when compared to wild type rats. No impairment in motor behavior was observed.

Gorbatyuk et al. [16] created three $\alpha$-syn models with the hWT $\alpha$-syn or mutated $\alpha$-syn with S129A, or S129D mutations. Unilateral injections of $\mathrm{rAVV} 2 / 5$ vectors expressing the transgenes were done in the SN. Histological examinations were carried out at 4,8 , or 26 weeks after injection. At 4 weeks, a $70 \%$ loss in TH-positive neurons was observed in the rats receiving S129A, whereas all other groups did not show a significant TH-positive neuronal loss, when compared to controls. At 8 weeks, rats receiving an injection of WT $\alpha$-syn rats exhibited a $40 \%$ loss of TH-positive neurons and S129A $\alpha$-syn rats exhibited a $66 \%$ loss of TH neurons. However, rats receiving hWT $\alpha$-syn exhibited a $60 \%$ loss of TH positive neurons at 27 weeks, which was similar to the S129A $\alpha$-syn rats at that time point. Rats receiving the S129D injections did not exhibit significant neurodegeneration at any of the time points. Striatal DA levels were assessed by HPLC. The HPLC analysis showed that there was a depletion of DA levels, consistent with SN cell loss.

Azeredo da Silveira et al. [17] created an $\alpha$-syn model to observe a prevention of the phosphorylation of human mutated $\alpha$-syn which is involved in numerous neurodegenerative diseases. The study utilized the rAAV2/6 vector with the CMV promoter. The site-directed mutations of human A30P $\alpha$-syn and hWT $\alpha$-syn were at the serine residue at position 129 . The serine residue was converted to alanine (S129A) to abolish phosphorylation or converted to aspartate (S129D) to reproduce effects of phosphorylation. 
The two site-directed mutations of the mutated human A30P $\alpha$-syn and hWT $\alpha$-syn were compared to hWT $\alpha$-syn and mutated human A30P $\alpha$-syn. Rats received two injections within the SN. They observed a dose-dependent loss from 11 to $22 \%$ in $\mathrm{TH}$-positive neurons when rats received injections of hWT $\alpha$-syn. Injection of the S129A-Mutated A30P $\alpha$ syn and hWT $\alpha$-syn resulted in a dose-dependent loss of over $70 \%$. Whereas the S129D-mutated A30P $\alpha$-syn and human WT $\alpha$-syn resulted in less neurodegeneration in the $\mathrm{SN}$ then WT $\alpha$-syn, rats receiving injections of hWT $\alpha$-syn with the mutated human A30P $\alpha$-syn tended to display less neurodegeneration than the WT $\alpha$-syn rats.

Finally, a series of $\alpha$-syn models of PD were produced by Lo Bianco et al. [14], using an HIV-1-derived lentivirus, expressing a range of $\alpha$-syn genes, including wild-type human $\alpha$-syn, mutated-human A30P $\alpha$-syn, mutated-human A53T $\alpha$-syn, and rat wild-type $\alpha$-syn. The largest reduction of TH-positive neurons within the $\mathrm{SN}$ was observed in animals treated with the lenti-WT human $\alpha$-syn, which exhibited a $35 \%$ reduction. Rats treated with A30P $\alpha$-syn and A53T $\alpha$ syn also exhibited a $33 \%$ and $24 \%$ reduction in TH-positive neurons, respectively. Some $\alpha$-syn inclusions were found in the cytoplasm of neurites and cell bodies of surviving nigral neurons.

Subsequent studies using genes that overexpressed glial derived neurotrophic factor (GDNF), a protein associated with neuroprotection of dopaminergic neurons, were unsuccessful in reducing the cell loss in several of these $\alpha$-syn rat models of PD. For example, Lo Bianco et al. [23] found that injections of a lenti-GDNF just dorsal to the SN, given two weeks prior to bilateral injections of lenti-A30P- $\alpha$-syn, failed to reduce the loss of DA neurons as assessed by TH immunohistochemistry. Similarly, Decressac et al. [24] injected lenti-GDNF into the striatum two weeks prior to an intranigral injection of rAAV2-A30P- $\alpha$-syn and, in a second study, injected rAAV2-GDNF in the striatum and just dorsal to the SN at three weeks prior to an intranigral injection of rAAV2-A30P- $\alpha$-syn and found that these GDNF injections failed to protect against $\alpha$-syn-induced neurotoxicity in both studies.

Lo Bianco et al. also tested the potential efficacy of using lenti-viral delivery of parkin in an $\alpha$-syn rat model of PD [25]. Parkin is a 465-amino-acid protein responsible for protein degradation, and mutated forms of parkin are found in about $50 \%$ of the cases of juvenile PD, an autosomal recessive form of this disease [26]. Lo Bianco et al. gave two groups of rats bilateral injections into the right $\mathrm{SN}$ of either lenti-A30P- $\alpha$-syn/lenti-YFP, or lenti-A30P- $\alpha$ syn/lenti-Parkin (with the Parkin gene being derived from a wild-type rat). Control animals received injections of either lenti-Parkin or lenti-YFP. A 31\% reduction of $\mathrm{TH}$-positive neurons in the $\mathrm{SN}$ was observed in animals receiving lentiA30P- $\alpha$-syn, whereas a $9 \%$ reduction in $\mathrm{TH}$ positive neurons was observed in the SN of animals receiving lenti-A30P- $\alpha$ syn with lenti-Parkin. There was a $16 \%$ reduction in $\mathrm{TH}-$ positive neurites within the striatum of the lenti-A30P- $\alpha$ syn rats. The animals receiving lenti-A30P- $\alpha$-syn with lentiParkin exhibited a $4 \%$ reduction in $\mathrm{TH}$-positive neurites within the striatum. In addition, animals receiving lentiA30P- $\alpha$-syn with lenti-Parkin, as evidenced by silver staining, did not show an $\alpha$-syn-induced neurodegeneration, whereas the lenti-A30P- $\alpha$-syn group did. Animals receiving lentiA30P- $\alpha$-syn with lenti-Parkin exhibited a $45 \%$ increase in hyperphosphorylated $\alpha$-syn inclusion, and lenti-A30P- $\alpha$-syn animals showed a $43 \%$ increase in hyperphosphorylated $\alpha$ syn inclusions. Similarly, Yamada et al. [4] examined the effects of Parkin on the rAAV $\alpha$-syn model of PD. They injected rAAV expressing $\alpha$-syn and rAAV parkin at the same time directly into SN and observed a decrease of cell death of DA neurons within the SN and the number of apomorphineinduced rotations, compared to rat injected with rAAV $\alpha$-syn alone. Clearly, the $\alpha$-syn rat models of PD provide many of the features that resemble symptoms of PD. However, most of the models produce a relatively limited cell loss, which provides a useful model of early PD but lacks the extensive cell loss and behavioral deficits to accurately mimic the later stages of this disease. Early therapeutic trials in these rat models have suggested that overexpression of the gene for parkin, but not GDNF, may reduce TH-positive cell loss in this model of PD.

\section{4-Leucine-Rich Repeat Kinase 2 (LRRK2)}

A second type of transgenic rat model involves the use of LRRK2, which is a member of the ROCO protein family and the RAS-GTPase super family. The LRRK2 gene contains both a GTPase-like domain and a kinase-like domain. An important number of mutations in the gene have been identified and associated with PD. Of these mutations, G2019S has been found to be the most common [27, 28] and is a point mutation located within the kinase domain. Another common mutation is R1441C, in which a mutation occurs within the GTPase domain.

Zhou et al. [21] utilized LRRK2 transgenic rats possessing the G2019S mutation. Transgenic rats were created by crossing TRE-LRRK2 G2019S transgenic rats with CAGtTA transgenic rats. These investigators examined the effects of temporal expression of the human LRRK2 mutation, using a promoter that is activated by the absence of doxycycline. They measured the total distance and stereotypical movements the rats made in an open-field maze during a 20-minute assessment period, followed by an addition 20-minute period, which was given 5 minutes after an injection of amphetamine or 20 minutes after an injection of nomifensine (an inhibitor of DA reuptake). They found that the temporal expression of the human LRRK2 G2019S increased the distance traveled as well as the amount of stereotypical behavior. When given nomifensine or amphetamine, the temporal expression of LRRK2 G2019S significantly reduced the amount of evoked responses, compared to controls. In addition to the openfield assessments, these investigators measured intrastriatal DA via microdialysis and found an increase in extracellular DA in the striatum of rats receiving temporal expression of LRRK2. However, there were no increase in the level of DA when amphetamine and nomifensine was administered 
to these rats. The researchers attributed these results to an impairment of the DA transporter (DAT) due to preservation of in $\mathrm{TH}$-positive neurons within the $\mathrm{SNpc}$, nor were there any changes observed in the number of axon terminals in the striatum.

Dusonchet et al. [20] produced a transgenic LRRK2 model in adult rats utilizing recombinant human serotype 5 adenovirus (RAd). These investigators compared the overexpression of human wild-type LRRK2 to the LRRK2 G2019S human mutation. They found no loss of TH-positive cells in the human wild-type LRRK2 animals. However, there was a progressive $\mathrm{TH}$-positive cell loss, observed between 10 and 42 days (with a total loss of $21.4 \%$ ), and a $10 \%$ decrease in striatal TH-positive fibers in G2019S LRRK2 animals.

As with the $\alpha$-syn models, the LRRK2 transgenic rat models of PD appear to provide only a modest loss of THpositive cells. However, there is evidence of motor deficits, as measured in the open-field maze, when the LRRK2 G2019S gene is activated in this transgenic rat model of PD. As such, this model may also provide a useful tool for evaluating potential treatments during the early stages of PD.

\section{Conclusion}

In this paper, we have provided an update on the various genetic rat models of PD. Although there is considerable variability among the studies reviewed in terms of the types of genes and techniques used, most of these models revealed a progressive and significant, albeit modest, loss of TH-positive neurons in the SNpc. As can be ascertained from Table 1, the $\alpha$-syn models show progressive cell loss, with protein aggregation, but little evidence for consistent motoric deficits. The use of the $\alpha$-syn A53T rat model did produce significant $\mathrm{TH}$-positive cell loss (at about 60\%) and concomitant motor deficits, but this occurred in only $25 \%$ of the rats receiving this mutated gene. The LRRK2 model produced both a level of cell loss comparable to earlystage PD and motor deficits in the open-field maze, but there is no evidence of protein aggregation in these animals. In terms of treatments, studies using transgenic PD rats have indicated that parkin can provide a significant level of protection against dopaminergic neuronal loss. Collectively, these studies indicate that the currently used transgenic rat models of PD can provide a useful tool for the early stages of PD, but more work is needed in developing a model that mimics the progressive neuropathology and behavioral deficits that are observed throughout the time span of this disease.

\section{Acknowledgments}

The authors gratefully acknowledge the generous support from the John G. Kulhavi Professorship (to GLD), the Field Neurosciences Institute, and the College of Humanities and Social and Behavioral Sciences at Central Michigan University.

\section{References}

[1] T. M. Dawson, "New animal models for Parkinson's disease," Cell, vol. 101, no. 2, pp. 115-118, 2000.

[2] M. G. Spillantini, M. L. Schmidt, V. M. Y. Lee, J. Q. Trojanowski, R. Jakes, and M. Goedert, " $\alpha$-synuclein in Lewy bodies," Nature, vol. 388, no. 6645, pp. 839-840, 1997.

[3] O. Cooper, P. Hallett, and O. Isacson, "Using stem cells and iPS cells to discover new treatments for Parkinson's disease," Parkinsonism \& Related Disorders, vol. 18, supplement 1, pp. S14-S16, 2012.

[4] M. Yamada, Y. Mizuno, and H. Mochizuki, "Parkin gene therapy for $\alpha$-synucleinopathy: a rat model of Parkinson's disease," Human Gene Therapy, vol. 16, no. 2, pp. 262-270, 2005.

[5] R. Jakes, M. G. Spillantini, and M. Goedert, "Identification of two distinct synucleins from human brain," FEBS Letters, vol. 345, no. 1, pp. 27-32, 1994.

[6] M. H. Polymeropoulos, J. J. Higgins, L. I. Golbe et al., "Mapping of a gene for Parkinson's disease to chromosome 4q21-q23," Science, vol. 274, no. 5290, pp. 1197-1199, 1996.

[7] M. H. Polymeropoulos, C. Lavedan, E. Leroy et al., "Mutation in the $\alpha$-synuclein gene identified in families with Parkinson's disease," Science, vol. 276, no. 5321, pp. 2045-2047, 1997.

[8] J. J. Zarranz, J. Alegre, J. C. Gómez-Esteban et al., “The new mutation, E46K, of $\alpha$-synuclein causes Parkinson and Lewy body dementia," Annals of Neurology, vol. 55, no. 2, pp. 164173, 2004.

[9] R. Krüger, W. Kuhn, T. Müller et al., "Ala30Pro mutation in the gene encoding $\alpha$-synuclein in Parkinson's disease," Nature Genetics, vol. 18, no. 2, pp. 106-108, 1998.

[10] R. G. Perez, J. C. Waymire, E. Lin, J. J. Liu, F. Guo, and M. J. Zigmond, "A role for $\alpha$-synuclein in the regulation of dopamine biosynthesis," Journal of Neuroscience, vol. 22, no. 8, pp. 3090-3099, 2002.

[11] F. J. S. Lee, F. Liu, Z. B. Pristupa, and H. B. Niznik, "Direct binding and functional coupling of $\alpha$-synuclein to the dopamine transporters accelerate dopamine-induced apoptosis," FASEB Journal, vol. 15, no. 6, pp. 916-926, 2001.

[12] D. Kirik, C. Rosenblad, C. Burger et al., "Parkinson-like neurodegeneration induced by targeted overexpression of $\alpha$ synuclein in the nigrostriatal system," Journal of Neuroscience, vol. 22, no. 7, pp. 2780-2791, 2002.

[13] R. L. Klein, M. A. King, M. E. Hamby, and E. M. Meyer, "Dopaminergic cell loss induced by human A30P $\alpha$-synuclein gene transfer to the rat substantia nigra," Human Gene Therapy, vol. 13, no. 5, pp. 605-612, 2002.

[14] C. Lo Bianco, J. L. Ridet, B. L. Schneider, N. Déglon, and P. Aebischer, " $\alpha$-synucleinopathy and selective dopaminergic neuron loss in a rat lentiviral-based model of Parkinson's disease," Proceedings of the National Academy of Sciences of the United States of America, vol. 99, no. 16, pp. 10813-10818, 2002.

[15] M. Yamada, T. Iwatsubo, Y. Mizuno, and H. Mochizuki, "Overexpression of $\alpha$-synuclein in rat substantia nigra results in loss of dopaminergic neurons, phosphorylation of $\alpha$-synuclein and activation of caspase-9: Resemblance to pathogenetic changes in Parkinson's disease," Journal of Neurochemistry, vol. 91, no. 2, pp. 451-461, 2004.

[16] O. S. Gorbatyuk, S. Li, L. F. Sullivan et al., "The phosphorylation state of Ser-129 in human $\alpha$-synuclein determines neurodegeneration in a rat model of Parkinson disease," Proceedings of the National Academy of Sciences of the United States of America, vol. 105, no. 2, pp. 763-768, 2008. 
[17] S. A. da Silveira, B. L. Schneider, C. Cifuentes-Diaz et al., "Phosphorylation does not prompt, nor prevent, the formation of $\alpha$-synuclein toxic species in a rat model of Parkinson's disease," Human Molecular Genetics, vol. 18, no. 5, pp. 872-887, 2009.

[18] A. Recchia, D. Rota, P. Debetto et al., "Generation of a $\alpha$ synuclein-based rat model of Parkinson's disease," Neurobiology of Disease, vol. 30, no. 1, pp. 8-18, 2008.

[19] F. Lelan, L. Lescaudron, C. Boyer et al., "Effects of human alpha-synuclein A53T-A30P mutations on SVZ and local olfactory bulb cell proliferation in a transgenic rat model of Parkinson disease," Parkinson's Disease, vol. 2011, Article ID 987084, 11 pages, 2011.

[20] J. Dusonchet, O. Kochubey, K. Stafa et al., "A rat model of progressive nigral neurodegeneration induced by the Parkinson's disease-associated G2019S mutation in LRRK2," Journal of Neuroscience, vol. 31, no. 3, pp. 907-912, 2011.

[21] H. Zhou, C. Huang, J. Tong, W. C. Hong, Y. -J. Liu, and X. -G. Xia, "Temporal expression of mutant LRRK2 in adult rats impairs dopamine reuptake," International Journal of Biological Sciences, vol. 7, no. 6, pp. 753-761, 2011.

[22] V. Sanchez-Guajardo, F. Febbraro, D. Kirik, and M. RomeroRamos, "Microglia acquire distinct activation profiles depending on the degree of $\alpha$-synuclein neuropathology in a rAAV based model of Parkinson's disease," PLoS One, vol. 5, no. 1, Article ID e8784, 2010.

[23] C. Lo Bianco, N. Déglon, W. Pralong, and P. Aebischer, "Lentiviral nigral delivery of GDNF does not prevent neurodegeneration in a genetic rat model of Parkinson's disease," Neurobiology of Disease, vol. 17, no. 2, pp. 283-289, 2004.

[24] M. Decressac, A. Ulusoy, B. Mattsson et al., "GDNF fails to exert neuroprotection in a rat $\alpha$-synuclein model of Parkinson's disease," Brain, vol. 134, no. 8, pp. 2302-2311, 2011.

[25] C. Lo Bianco, B. L. Schneider, M. Bauer et al., "Lentiviral vector delivery of parkin prevents dopaminergic degeneration in an $\alpha$-synuclein rat model of Parkinson's disease," Proceedings of the National Academy of Sciences of the United States of America, vol. 101, no. 50, pp. 17510-17515, 2004.

[26] T. Kitada, S. Asakawa, N. Hattori et al., "Mutations in the parkin gene cause autosomal recessive juvenile parkinsonism," Nature, vol. 392, no. 6676, pp. 605-608, 1998.

[27] J. P. Taylor, I. F. Mata, and M. J. Farrer, "LRRK2: a common pathway for parkinsonism, pathogenesis and prevention?" Trends in Molecular Medicine, vol. 12, no. 2, pp. 76-82, 2006.

[28] I. F. Mata, O. A. Ross, J. Kachergus et al., "LRRK2 mutations are a common cause of Parkinson's disease in Spain," European Journal of Neurology, vol. 13, no. 4, pp. 391-394, 2006. 


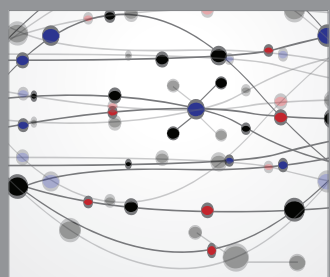

The Scientific World Journal
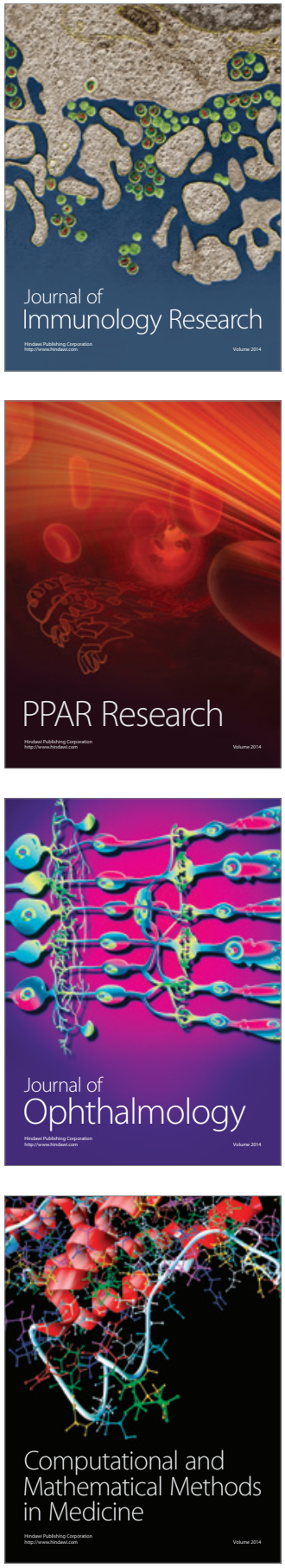

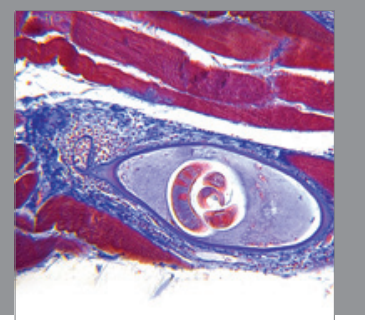

Gastroenterology

Research and Practice
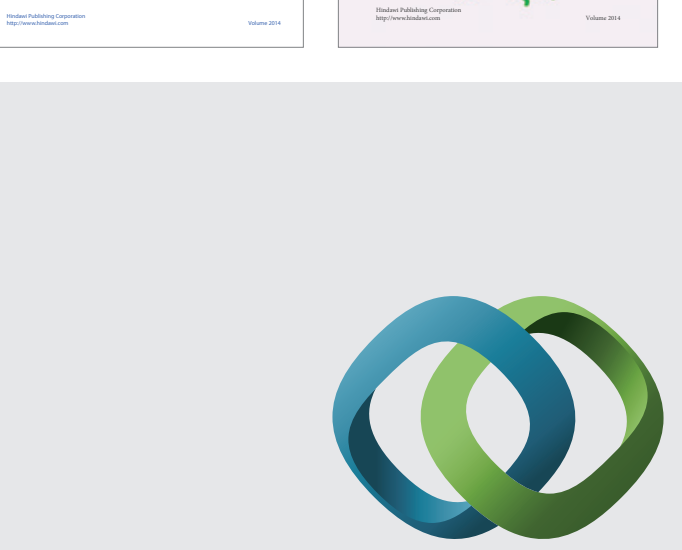

\section{Hindawi}

Submit your manuscripts at

http://www.hindawi.com
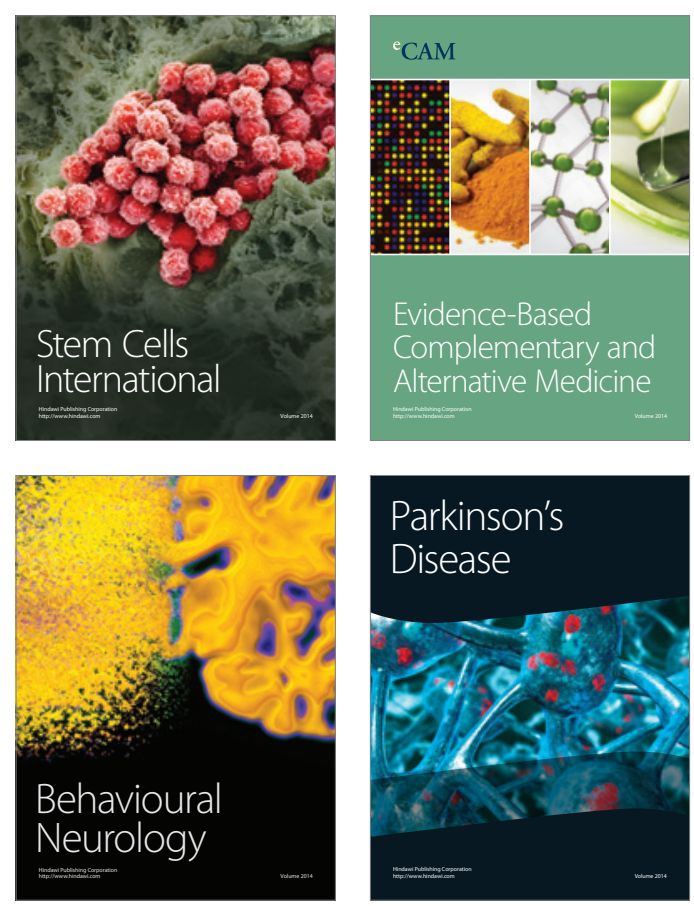

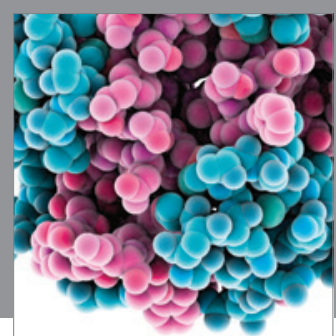

Journal of
Diabetes Research

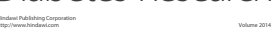

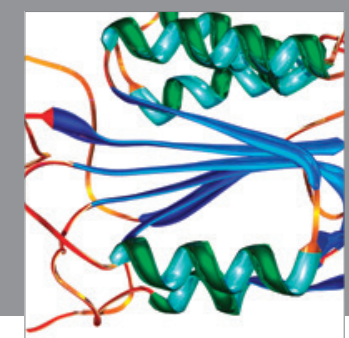

Disease Markers
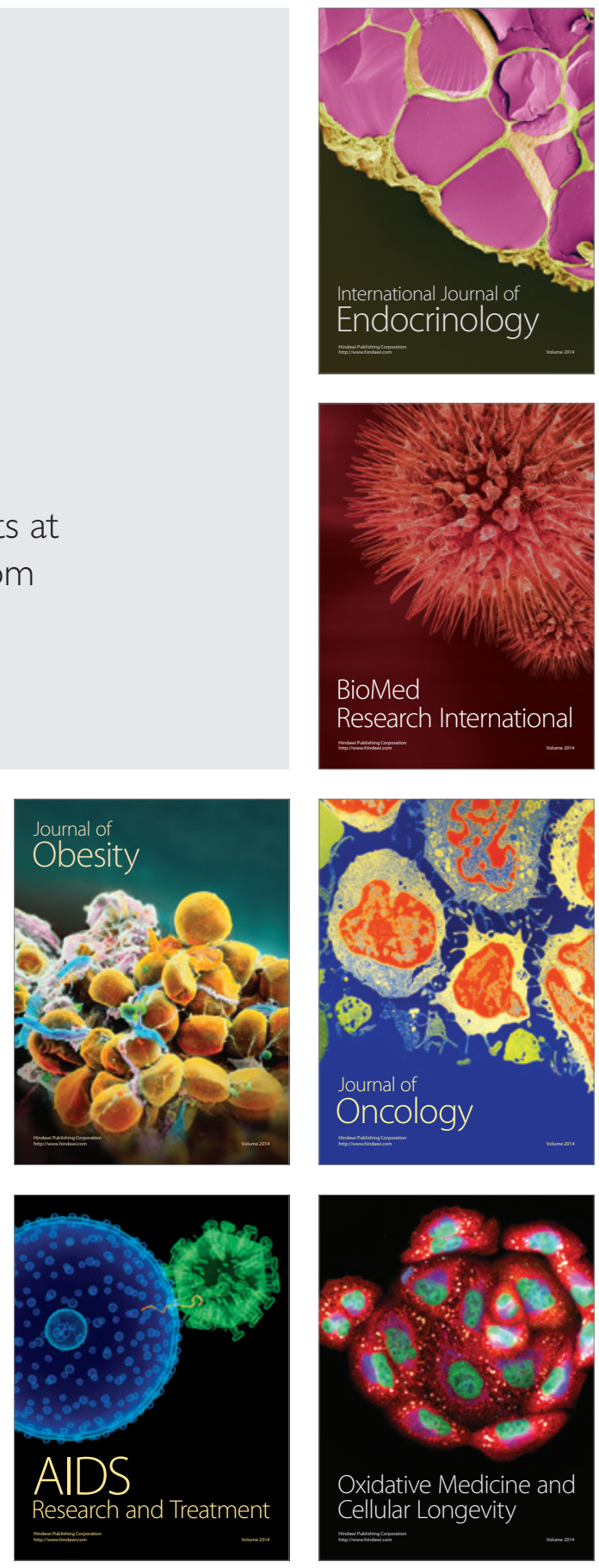